\title{
Enterprise Information Integration
}

\author{
Nacira Ghoualmi-Zine \\ Computer Science Department \\ Badji Mokhtar University - Annaba \\ Annaba, Algeria \\ ghoualmi@yahoo.fr
}

\begin{abstract}
In this article, we aim to develop a Web application for a chemist industrial environment. This application provides interfaces for operator and interfaces for supervisor. All interfaces are included in MES (Manufacturing Execution System) functionalities. The first are used to collect information and so provide e-collection. The second are used for passive e-supervision. These last, are similar to synoptic but have not the same objectives. Interfaces are based on click on boxes. Stereotypes of EWA-UML are adequate for this application. Application is developed upon JSP technologies.
\end{abstract}

\section{Introduction}

We develop this application in Algerian chemist industrial environment. The factory produces ammoniac. It has DCS (Distributed Control System) that supervise production: NINA System I/A Series de FOXBORO. Exchange system is based on ETHERNET TCP/IP with server for history and five stations for control. In recent years MES have managed all activities and resources production processes in an effort to reduce cycle time, low inventory, and increase productivity [1]. Manufacturing Execution Systems (MES) are a recently defined category of industrial software for the plant floor/manufacturing environment. So, our work is integrated in MES and has two objectives: First, reduce time for data collection and provide e-collection at plant floor. Second, provide passive supervision for agent at third level in CIM. This allows him to access database and display the most important information. In each case, we present interfaces.

Paper is organised as: In section one, we give an introduction. Section two we summarise developed applications. Section three presents interactions techniques. Section four presents component diagram of board journal. In section 5 we give architecture's application. In section 6 we present e-collection and e-supervision interfaces. Paper is ended by a conclusion. 


\section{Data Collection}

We note that some examples of applications that have been developed in areas such as general manufacturing [2], power generation [3], automotive [5], and electronics [4], [6] based on screen and GUI. For our work, we are interested in electronics collection.

\section{Interaction Techniques}

There are several software interaction techniques [7]. GUI was designed for interaction techniques that let the operators select elements that accomplish the desired tasks. Command buttons, and dialog boxes were some of the interaction techniques used for application to keep it simple in code design and ensure application usability. Buttons are individual and isolated regions within the display which can be selected by the operators to invoke specific operations. Clicking on the button invokes a command and its meaning is indicated by the button caption or button background colour. Dialog boxes are information windows used to capture the operators attention about some important information or giving feedback in the interaction.

\section{UML/WAE Design}

Journal board is the most important document. It contains whole data about production. It was a form filled by operator. Fig. 1 presents component diagram [8].

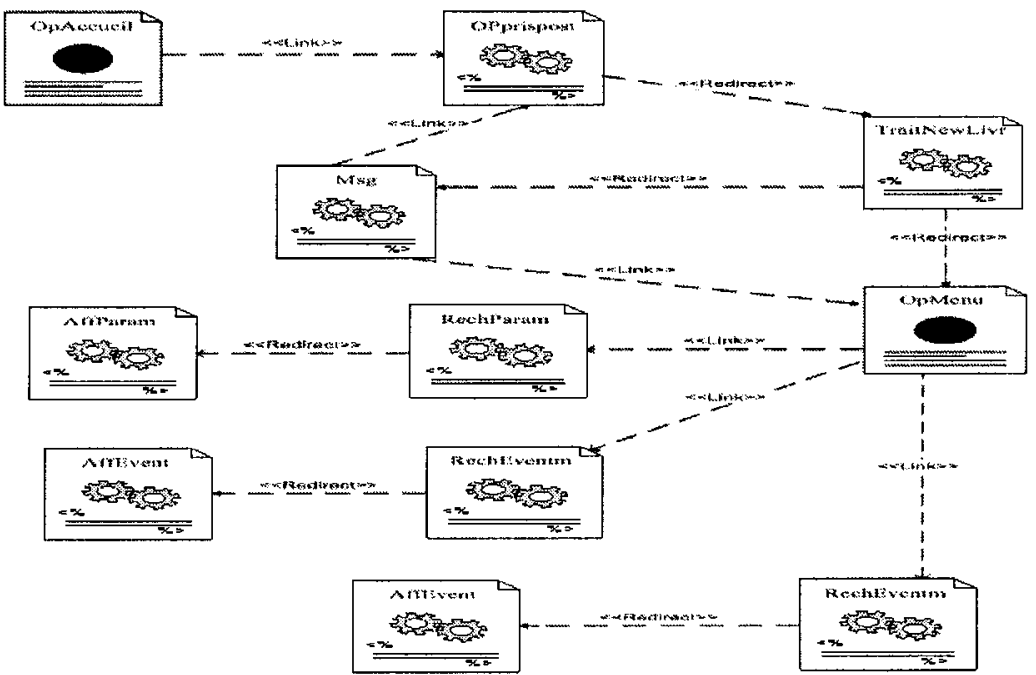

Fig. 1. Component diagram 


\section{Architecture System}

The SGRDB is Access 2002 and Requests are executed with JBDC/SQL. Application is deployed in 3-Tiers Client/server architecture based on JSP technologies and Javabean [9].

\section{Interfaces}

We present two types of interfaces: e-collection and e-supervision. All interfaces are in French language because it is the language used in Algeria, and all documents in factory are in French.

\section{1 e-collection}

Fig. 2 shows interface used to input event at the first floor of pyramid CIM. It is filled by operator.

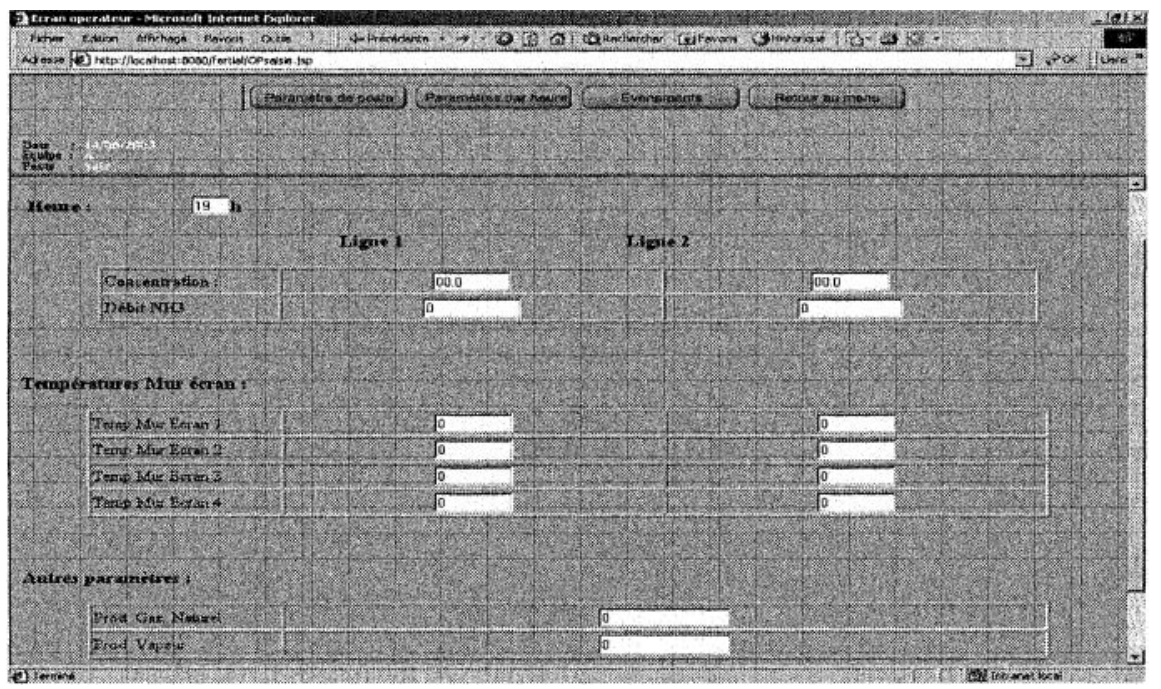

Fig. 2. Interface for event e-collection

Fig. 3 shows interface with form filled by operator to input important consummation. 


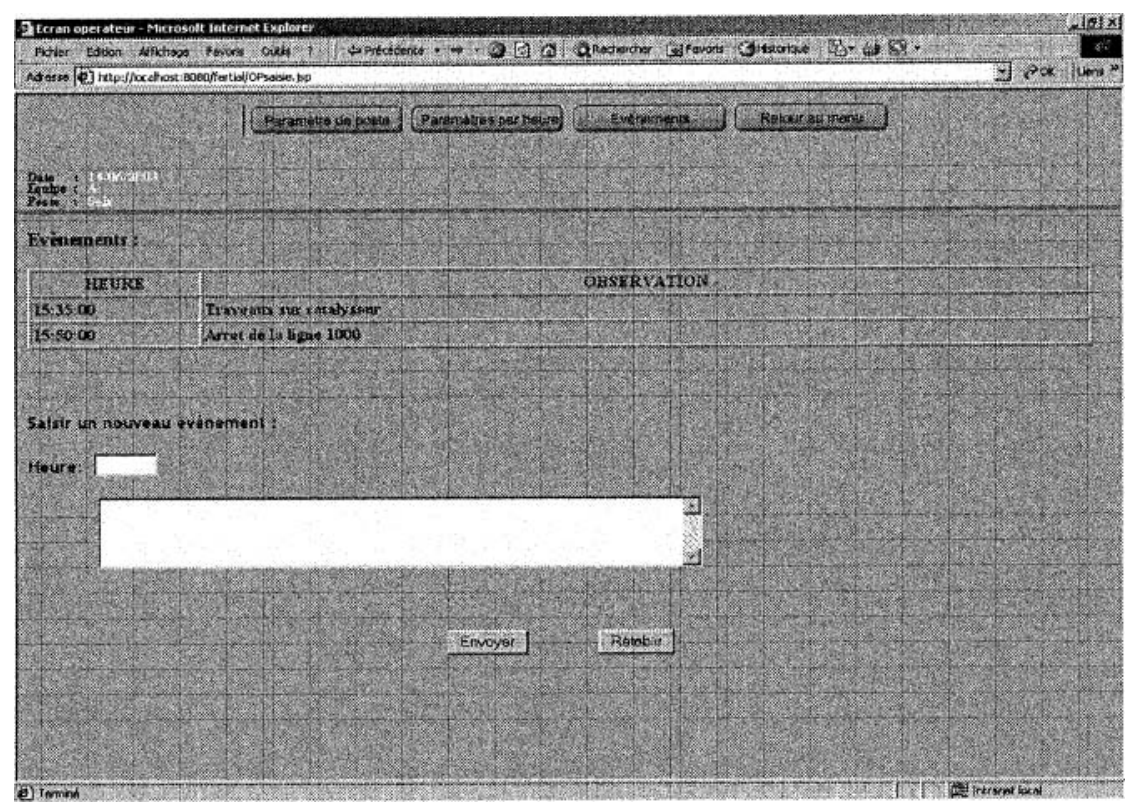

Fig.3. Interface for e-collection

\subsection{Passive e-Supervision}

Interface for passive e-supervision is similar to synoptic developed for floor plant in production of NHO3. It provides a real vision on plant and it is used by agent to access at data in data base. Agent clicks on green box to display data associated to consummation of natural gas (in middle of interface) and associated to consummation of vapour (in bottom of interface). 


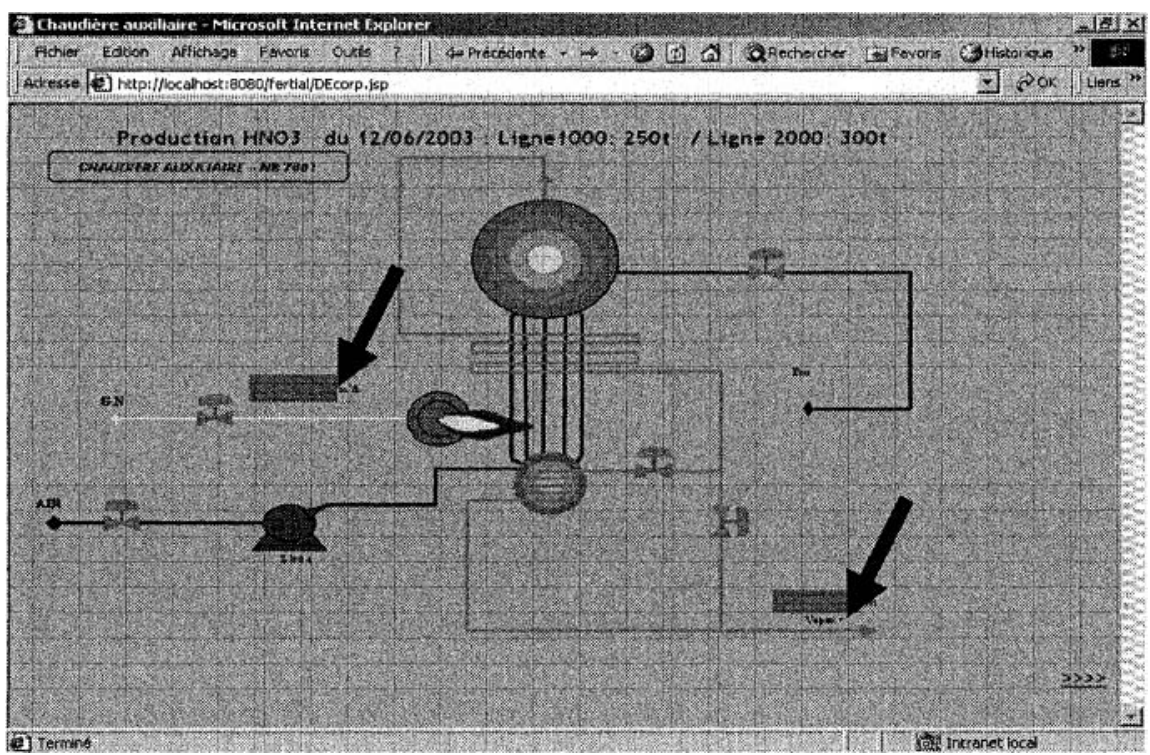

Fig. 4. Supervision interface for $\mathrm{NHO3}$ production

Fig. 4 is similar to synoptic developed for floor plant in production of NHO3. It provides a real vision on plant and it is used by agent in third floor to access at data in data base. Agent clicks on green box to display data associated to consummation of FI103 (in middle of interface) and associated to debit of $\mathrm{NH} 3$ (in middle of interface).

\section{Conclusion}

In this paper we have developed enterprise information system integration with two types of interfaces: the first for operator to collect information and store them in real time. The second for agent in third floor of pyramid CIM. These interfaces are similar to synoptic but have not the same role. It indicates by click on boxes to the agent the values of most important parameters. We used EWA-UML for conception. This application reduces document and storage time and provides a real vision for agent when he access to production parameters. However, this application must have a high security to avoid intrusions because it is installed over a TCP/IP.

\section{References}

1. E.J. Byres, Designing Secure Networks for Process Control, IEEE Industry Applications Magazine September /October, 33-35 (2000). 
2. M. Bigam, Made2 Manage Systems Adds Wireless Shop Floor Data Collection to Improve Operational Efficiency and Back-End System Intelligence, Managing Automation Online (2005), available from: http://www.managingautomation.

3. R.P. Klump, et al., Real-Time Data Retrieval and New Visualization Techniques for the Energy Industry, IEEE Computer Society Proceedings of the $35^{\text {th }}$ International Conference on Systems Sciences, September 2002.

4. T. Luoh, L. Yang, H. Shih, C. Huang, K. Chen, Y. Hwang, and C. Hsueh, and H. Chung, Process Optimization and Productivity Improvement by Real-Time Data Collection System, Semiconductor Manufacturing Technology Workshop, 8, July 2002, pp. 140-143.

5. P. Ramanathan, Overload Management in Real-Time Control Applications Using (m,k) Firm Guarantee, IEEE Transactions on Parallel and Distributive Systems 10(6), 549-559 (1999).

6. K. Preston, C. Mastrangelo, and F. Anastasio, and B. Best, Reenginering the Operator Interface for a Semiconductor Manufacturing Execution System, IEEE International Conference on Systems, Man, and Cybernetics, 3, December 2000, pp 1799-1804.

7. B. Grattan, G. Stitt and F. Vahid, Codesign-Extended Application, IEEE/ACM International Symposium on Hardware/Software Codesign, Estes Park, May 2002, pp. 1-6.

8. J, Conallen, Concevoir des Applications Web avec UML, Editions Eyrolles, 2000.

9. L. Lemau, and R. Cadenhead, Le Programmeur : Java 2 Plate-Forme (Campus Press, France, 2000). 